\title{
Castration Resistance
}

National Cancer Institute

\section{Source}

National Cancer Institute. Castration Resistance. NCI Thesaurus. Code C130235.

A finding indicating that a prostate carcinoma continues to grow despite the surgical removal of the testes or medical intervention to block androgen production. 